\title{
Mensuração do tamanho cardíaco pelo método VHS (vertebral heart size) em cães sadios da raça American pit bull terrier
}

\author{
Measurement of heart size by VHS (vertebral heart size) method in healthy American pit bull terrier
}

\author{
Mauro José Lahm Cardoso I Jonatas Luiz Caludino"I Maíra Melussi'II
}

RESUMO

\begin{abstract}
Vários métodos surgiram com a finalidade de aumentar a acurácia e diminuir a subjetividade na avaliação das radiografias. $O$ melhor aceito é o VHS (vertebral heart size), ou tamanho do coração em relação à unidade de vértebra torácica. O objetivo deste trabalho foi aplicar o método de mensuração pelo VHS em cães da raça American pit bull terrier clinicamente sadios, com a finalidade de se estabelecer o valor médio de VHS para esta raça. Realizaram-se radiografias de tórax, na projeção lateral direita, que foram avaliadas empiricamente e realizadas as medidas necessárias para obtenção do VHS, além da obtenção da relação profundidade/largura $(P / L)$ torácica. Nenhum animal apresentou alteração através da análise empírica. Os valores de VHS obtidos apresentaram distribuição normal, assim como a relação $P / L$, com média de $10,9 \pm 0,4$ vértebras para o VHS e $0,80 \pm 0,07$ para a relação $P / L$. O valor do VHS apresentou diferença significativa $(P>0,05)$ em comparação com os resultados obtidos por BUCHANAN \& BÜCHELER (1995). Porém, o valor de VHS deste estudo é semelhante ao descrito por diversos autores nos estudo de raças especificas, confirmando a necessidade de se estabelecer valores de VHS específicos para cada raça.
\end{abstract}

Palavras-chave: coração, radiografia torácica, cães.

\section{ABSTRACT}

In order to increase accuracy and reduce the subjectivity in the evaluation of radiographies, several methods have emerged. The most accepted of these methods is the Vertebral Heart Size method (VHS), or the heart's size versus the thoracic vertebra's unit. The intention of this research was to apply the VHS measurement method in clinically healthy
American pit bull terrier, with the purpose of establishing the average VHS for this breed. Chest x-rays in lateral projection were taken, and these were empirically evaluated and measured so that a VHS value was obtained, as well as the thoracic depth/ width $(D / W)$ relation for each animal. None of the animals had abnormal results when evaluated via empiric analysis. VHS values had a normal distribution, as did $D / W$ relations, with an average of 10.9 $\pm 0,4$ vertebrae for VHS and $0.80 \pm 0,07$ for $D /$ $W$ relations. VHS values showed a significant difference $(P>0.05)$ when compared to the results obtained by BUCHANAN \& $B \ddot{U C H E L E R ~(1995) . ~ H o w e v e r, ~ V H S ~ v a l u e s ~ i n ~ t h i s ~ s t u d y ~ a r e ~}$ similar to those described by several other authors, confirming the need to establish values of VHS for each breed.

Key words: heart, chest radiography, dog.

\section{INTRODUÇÃO}

O exame radiográfico do tórax fornece subsídios essenciais para a avaliação e diagnóstico de doenças ou alterações cardíacas primárias e secundárias. As radiografias torácicas também podem sugerir o prognóstico e a terapia a ser instituída, servindo como meio de acompanhamento da evolução da doença (BUCHANAN \& BÜCHELER, 1995; ROOT \& BAHR, 2002). É importante ressaltar que o diagnóstico das doenças cardíacas não é baseado somente nos achados radiográficos, sendo necessário associar sinais clínicos, achados físicos e também exame eletrocardiográfico e ecocardiográfico (LAMB et al., 2000).

'Hospital Veterinário, Universidade Estadual do Norte do Paraná (UENP), campus Luiz Meneghel (CLM), Bandeirantes, PR, BR 369, Km 54, CP 261, 86360-000, Bandeirantes, PR, Brasil. E-mail: maurolahm@uenp.edu.br. Autor para correspondência.

"UENP, CLM, Bandeirantes, PR, Brasil.

II'Médica Veterinária Autônoma, Londrina, PR, Brasil. 
Vários estudos foram realizados objetivando aumentar a acurácia e diminuir a subjetividade do estudo radiográfico da silhueta cardíaca. Dessa forma, surgiram novos métodos quantitativos e, dentre eles, a proposta melhor aceita foi a realizada por BUCHANAN \& BÜCHELER (1995). Esse método é denominado VHS (vertebral heart size), ou avaliação tamanho do coração em relação à unidade de vértebra torácica, e compara as dimensões cardíacas aos comprimentos das vértebras torácicas.

As vantagens apresentadas pelos métodos radiográficos de mensuração da silhueta cardíaca são rapidez, praticidade e facilidade de reprodução, independente do grau de experiência do profissional, tanto no que se refere à triagem dos animais como sendo portadores de cardiomegalia, quanto no acompanhamento do tratamento instituído e evolução da doença (LORD, 1974; BUCHANAN \& BÜCHELER, 1995; SOARES et al., 2004).

Para avaliação radiográfica da silhueta cardíaca é imprescindível o reconhecimento das variações normais relacionadas ao tamanho e posição da imagem cardíaca, de acordo com a conformação do tórax e idade do animal. A grande variedade de conformações torácicas observadas nas raças de cães é um dos fatores que interfere na análise comparativa da silhueta cardíaca, uma vez que o coração sofre mudanças em seu tamanho e posicionamento na dependência das dimensões do tórax do animal (BUCHANAN e BÜCHELER, 1995). Segundo LAMB et al. (2001) e GÜLANBER et al. (2005), existem diferenças significativas entre os valores de VHS para as diferentes raças, o que torna imprescindível a padronização desses valores para cada raça.

De acordo com SOARES et al. (2004), há correlação positiva entre a evolução dos sinais clínicos de cardiopatia e a medida do VHS na avaliação cardíaca de cães de diversas raças, nas diferentes classes funcionais de insuficiência cardíaca, segundo o critério descrito pelo International Small Animal Cardiac Health Council (ISACHC). Esses resultados mostram a importância clínica desse método de avaliação cardíaca.

GUGLIELMINE et al. (2009) obtiveram resultados diferentes de VHS para animais com tosse de origem cardíaca $(12,8 \pm 1)$ e de origem não cardíaca $(11 \pm 0,9)$. A tosse é um sinal clínico frequente de doença cardiovascular em cães. É causada pelo aumento atrial que leva à compressão brônquica, mas também pode estar presente em distúrbios respiratórios. O VHS foi útil na avaliação da progressão do aumento cardíaco em sete casos de insuficiência de valva mitral adquirida, durante um período de 3,5 anos (BUCHANAN \& BÜCHELER, 1995).
O objetivo deste trabalho foi aplicar o método proposto por BUCHANAN \& BÜCHELER (1995) em cães da raça American pit bull terrier clinicamente saudáveis, com a finalidade de estabelecer o valor médio de VHS para esta raça amplamente difundida em nosso meio.

\section{MATERIAL E MÉTODOS}

Foram utilizados nesta pesquisa 20 cães sadios da raça American pit bull terrier, pertencentes a clientes do Hospital Veterinário da Universidade Estadual do Norte do Paraná (UENP), campus de Bandeirantes (PR). Foram avaliados 12 machos $(60 \%)$ e 8 fêmeas $(40 \%)$, com idade variando de um a seis anos (média de três anos) e pesando em média $29,29 \mathrm{~kg} \pm 6,09$. Foram excluídos do estudo animais com alterações cardiovasculares. Para exclusão dos animais portadores de doenças cardiovasculares foram realizados exame físico, eletrocardiograma e análise empírica das radiografias, a avaliação empírica também foi útil para análise inicial das radiografias.

Posteriormente, obteve-se a mensuração do VHS nos animais sadios através do método proposto por BUCHANAN \& BÜCHELER (1995). As medidas foram obtidas através de radiografias laterais direita. $\mathrm{O}$ eixo longo do coração (L) foi mensurado desde o bordo ventral do centro do brônquio principal esquerdo ao contorno mais distante do ápice cardíaco. Essa medida foi reposicionada sobre as vértebras torácicas, com início sobre a borda cranial de $\mathrm{T} 4$, e convertida em unidade de vértebra com a aproximação de 0,1 vértebra (Figura 1).

Da mesma radiografia em que se obteve o eixo longo, o eixo curto (S) foi obtido no terço médio cardíaco, perpendicular ao eixo longo e transformado em unidade de vértebra a partir da borda cranial de T4, assim como no eixo longo (Figura 1). Para se obter o VHS, as dimensões do eixo longo (L) e curto (S) foram então somadas como descrito por BUCHANAN \& BÜCHELER(1995).

A relação profundidade/largura $(\mathrm{P} / \mathrm{L})$ do tórax foi realizada a fim de determinar se o tórax é profundo ou largo. A profundidade do tórax foi obtida na radiografia lateral, mensurando a distância entre $o$ bordo cranial do processo xifóide e o bordo ventral vertebral. O comprimento torácico foi mensurado em projeção ventrodorsal (VD), na distância entre os bordos mediais da oitava costela, na curvatura lateral maior (FONSECA PINTO \& IWASAKI 2002).

A análise estatística foi obtida pelo teste $\mathrm{T}$ com amostras independentes para o resultado do VHS. Foi considerado significativo $\mathrm{P}<0,05$. Compararam-se 


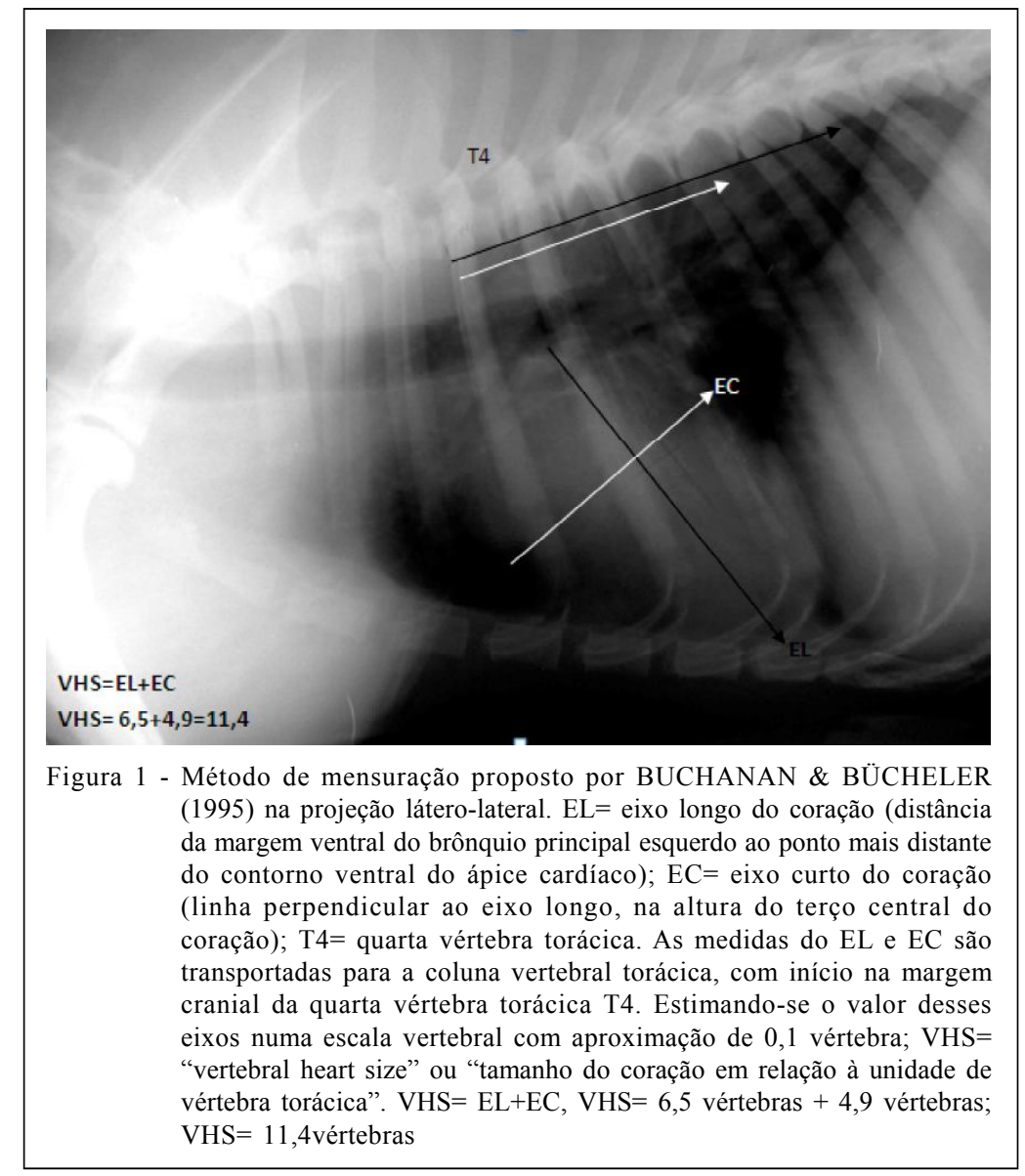

os resultados do VHS obtidos neste trabalho com os valores obtidos de várias raças por BUCHANAN \& BUCHELER(1995).

\section{RESULTADOS}

A análise subjetiva das radiografias não mostrou alteração nas câmaras cardíacas. Apenas três animais (15\%) apresentaram medidas menores ou iguais a 10,6 vértebras. A média dos valores de VHS foi de $10,9 \pm 0,4$ vértebras, sendo o valor mínimo encontrado igual a 10,5 vértebras e o máximo igual a 11,8, sem diferença significativa entre os cães. Os resultados na comparação entre os sexos estão distribuídos na tabela 1.

Os valores do VHS em cães da raça American pit bull terrier apresentaram diferença significativa $(\mathrm{P}>0,05)$ em comparação com os resultados obtidos por BUCHANAN \& BÜCHELER (1995). A média da relação entre profundidade e largura (P/L) foi de $0,80 \pm 0,07$.

\section{DISCUSSÃO}

O VHS é um método de fácil aplicação e objetivo para determinar as dimensões do coração na prática clínica (GÜLANBER et al., 2005). NAKAYAMA et al. (2001) fizeram estudos eletrocardiográficos e ecocardiográficos, comparando o tamanho do coração com o método VHS, no qual observaram resultados similares entre os métodos para cães com cardiomegalia. As doenças cardíacas geralmente cursam com cardiomegalia, portanto, são identificadas na radiografia lateral pelo método de VHS ou por avaliação radiográfica subjetiva. Porém, nem sempre a doença cardíaca se manifestará pelo aumento do coração, consequentemente pode-se lançar mão de outros métodos diagnósticos como a ecocardiografia e a tomografia computadorizada (LAMB \& BOSWOOD, 2002).

Para mensuração do VHS, foram realizadas apenas radiografias laterais direita, como método de padronização destas, apesar de alguns autores não 
Tabela 1 - Médias, desvios-padrão e intervalos da mensuração do tamanho cardíaco pelo método VHS (vertebral heart size) em 20 cães sadios da raça American pit bull terrier.

\begin{tabular}{lccc}
\hline & Média & Desvio Padrão & Intervalo \\
\hline Fêmeas (8) & $10,94 \mathrm{v}^{\mathrm{a}}$ & $0,34 \mathrm{v}$ & $10,5-11,4 \mathrm{v}$ \\
Machos (12) & $10,99 \mathrm{v}^{\mathrm{a}}$ & $0,33 \mathrm{v}$ & $10,6-11,8 \mathrm{v}$ \\
Total (20) & $10,97 \mathrm{v}^{\mathrm{a}}$ & $0,33 \mathrm{v}$ & $10,5-11,8 \mathrm{v}$ \\
\hline
\end{tabular}

Valores seguidos de mesmas letras não diferem entre si $(\mathrm{P}>0,05)$. $\mathrm{v}=$ vértebras.

encontrarem diferenças significativas nos valores de VHS na mensuração lateral em relação à ventrodorsal, como também entre as projeções laterais direita e esquerda (BUCHANAN \& BÜCHELER, 1995). Outros, no entanto, obtiveram valores significativamente maiores em decúbito lateral direito, em comparação com o esquerdo. É importante salientar que, nesses animais, o sexo e o tamanho do animal não teve influência sobre os valores de VHS (GRECO et al., 2008). Pode-se atribuir pequenas variações nas medidas do VHS entre radiografias esquerdas e direitas, devido a diferentes fases do ciclo cardíaco (TOAL et al., 1985) e do ciclo respiratório (SILVERMAN \& SUTER, 1975).

Na prática clínica, o posicionamento radiográfico lateral pode ser preferível ao posicionamento ventrodorsal, pois o primeiro é mais confortável e causa menos esforço ao paciente com suspeita de doença cardíaca (GÜLANBER et al., 2005).

Foram utilizados animais com idade entre um e seis anos, com base nos estudos realizados por HOLZMANN (1953) em humanos. O autor verificou que o coração de crianças recém-nascidas é maior proporcionalmente em relação ao seu corpo, diminuindo até chegar às proporções de adultos aos três anos de idade. Segundo HARPSTER (1987), isso ocorre também com gatos neonatos, já que a relação é de $9,3 \mathrm{~g} \mathrm{~kg}^{-1} \mathrm{e}$ cai para $4,0 \mathrm{~g} \mathrm{~kg}^{-1}$ quando adultos. É provável que ocorra também nos cães, o que poderia alterar os valores de VHS em animais não adultos. Porém, SLEEPER \& BUCHANAN (2001) e GÜLANBER et al. (2005), usando o VHS, não encontraram diferenças significativas entre os valores de cães neonatos dos 3 aos 36 meses de idade.

FONSECA PINTO \& IWASAKI (2002) avaliaram e compararam vários métodos radiográficos para identificação de aumento cardíaco e concluíram que o método proposto por BUCHANAN \& BÜCHELER (1995) é de fácil aplicação. O VHS médio nas raças cocker spaniel e boxer foi maior que 10,5 vértebras (v), sugerida por BUCHANAN \&
BÜCHELER (1995) como o limite superior para o tamanho da silhueta cardíaca, confirmando a necessidade de se padronizar valores de VHS nas diferentes raças (FONSECAPINTO \& IWASAKI, 2002).

Nos cães da raça American pit bull terrier, clinicamente normais deste estudo, com idade variando de um a seis anos, apresentaram valores médios de VHS de 10,9 90,4 vértebras. Esses valores apontaram diferença significativa $(\mathrm{P}>0,05)$ em comparação com os resultados obtidos por BUCHANAN \& BÜCHELER (1995). Porém, o valor de VHS deste estudo se altera de modo semelhante ao descrito em diversas raças como boxers $(11,6 \pm 0,8)$, cavalier king Charles spaniel $(10,6 \pm 0,5)$, labrador retriever $(10,8 \pm 0,6)$, whippets $(11,0 \pm 0,5)$ e greyhounds $(10,5 \pm 0,1)$ (LAMB et al. 2001 ; BEVEGEMS et al., 2005; MARIN et al., 2007). A média do VHS obtido de 10,9 vértebras é superior ao considerado como limite para a maioria das raças (VHS<10,5) (BUCHANAN \& BÜCHELER, 1995).

Neste trabalho, a relação profundidade/ largura $(\mathrm{P} / \mathrm{L})$ do tórax foi realizada para determinar se $\mathrm{o}$ tórax é largo ou profundo. A relação $\mathrm{P} / \mathrm{L}$ obtida foi de 0,8 e sabe-se que cães com relação $\mathrm{P} / \mathrm{L}=1,25 \mathrm{e}=0,75$ são classificados como animais de tórax profundo e longo, respectivamente. Nos cães com tórax curto, como os schnauzers, admite-se VHS > que 11 vértebras, assim como nos animais de tórax longo, como os dachshunds, admite-se VHS < que 9,5 vértebras (FONSECA PINTO \& IWASAKI 2002). Portanto, os cães da raça American pit bull terrier avaliados neste estudo possuem tórax com característica intermediária. Esse resultado não justifica os valores de VHS superiores ou iguais a 11 vértebras, observados em $45 \%$ dos cães.

A grande massa muscular dos cães da raça American pit bull terrier pode ser a melhor explicação para as possíveis causas do alto valor do VHS, quando comparado à outras raças. A necessidade do alto débito cardíaco para suprir a musculatura provocaria aumento na massa muscular cardíaca e, consequentemente, o coração seria relativamente maior que as outras raças de cães.

Com base neste trabalho, sugerimos, como limite inferior e superior de VHS para cães da raça American pit bull terrier, 10,5 e 11,8 vértebras, respectivamente. Este estudo demonstra a importância da padronização do VHS em diferentes raças, pois os valores aqui encontrados são superiores aos descritos como normal em estudos anteriores. 


\section{CONCLUSÃO}

Os resultados de VHS obtidos para os cães da raça American pit bull terrier foram: média 10,9 vértebras $( \pm 0,4)$, valor mínimo igual a 10,5 e máximo igual a 11,8 vértebras.

\section{AGRADECIMENTOS}

Este projeto foi realizado graças ao apoio do programa de iniciação científica $\mathrm{CNPq} / \mathrm{PIBIC}-$ UENP.

\section{COMITÊ DE ÉTICA}

Foi aprovado pelo Comitê em Ética o Uso de Animais (CEUA) da UENP.

\section{REFERÊNCIAS}

BEVEGEMS, V. et al. Vertebral heart size ranges specific for whippets. Veterinary Radiology and Ultrasound, v.46, p.400403, 2005. Disponível em: <http://onlinelibrary.wiley.com/doi/ 10.1111/j.1740-8261.2005.00073.x/pdf>. Acesso em: 17 ago. 2009. doi: $10.1111 / \mathrm{j} .1740-8261.2005 .00073 . x$.

BUCHANAN, J.W.; BÜCHELER, J. Vertebral scale system to measure canine heart size in radiographs. Journal of the American Veterinary Medical Association, v.206, n.2, p.194-199, 1995.

FONSECA PINTO, A.C.B.C.; IWASAKI, M. Métodos radiográficos de avaliação da silhueta cardíaca em cães. Veterinária Notícias, v.8, n.1, p.67-75, 2002.

GRECO, A. et al. Effect of left vs. right recumbency on the vertebral heart score in normal dogs. Veterinary Radiology \& Ultrasound, v.49, n.5, p.454-455, 2008. Disponível em: $<$ http://onlinelibrary.wiley.com/doi/10.1111/j.17408261.2008.00406.x/pdf>. Acesso: 17 ago. 2009. doi:10.1111/ j.1740-8261.2008.00406.x.

GUGLIELMINE, C. et al. Use of the vertebral heart score in coughing dogs with chronic degenerative mitral valve disease. Journal of Veterinary Medical Science, v.71, n.1. p.9-13, 2009. Disponível em: <http://www.jstage.jst.go.jp/article/jvms/ 71/1/71_9/_article>. Acesso em: 13 set. 2010. doi:10.1292/ jvms.71.9.

GÜLANBER, R.G. et al. Vertebral scale system to measure heart size in thoracic radiographs of Turkish Shepherd (Kangal) dogs. Turk Journal Veterinary Animal Science, v.29, p.723-726, 2005.

HARPSTER, N.K.; ZOOK, B.C. The cardiovascular system. In HOLZWORTH, J. (Ed.). Diseases of the cat: medicine and surgery. Philadelphia: Saunders, 1987. p.820.
HOLZMANN, M. Diseases of the heart and blood vessels. In: SCHINZ, H.R. et al. (Eds.). Roentgen diagnostics. New York: Grune \& Stratton, 1953. V.3, p.2819.

LAMB, C.R.; BOSWOOD, A. Role of survey radiography in diagnosing canine cardiac disease. Compendium on Continuing Education for the Practicing Veterinarian, v.24, p.316-326, 2002.

LAMB, C.R. et al. Assessment of the value of the vertebral heart scale in the radiographic diagnosis of cardiac disease in dogs. Veterinary Record, v.146, p.687-690, 2000.

LAMB, C.R. et al. Use of breed-specific ranges for the vertebral scale as an aid to the radiographic diagnosis of cardiac diseases in dogs. Journal of the American Veterinary Medical Association, v.230, p.1870-1876. 2001.

LORD, P.F. Cardiac mensuration. In: KIRK, R.W. Current veterinary therapy. Philadelphia: Saunders, 1974. p.339340 .

MARIN, L.M. et al. Vertebral heart size in retired racing greyhounds. Veterinary Radiology and Ultrassound, v.48, p.332-334. 2007. Disponível em: <http://onlinelibrary.wiley.com/ doi/10.1111/j.1740-8261.2007.00252.x/pdf>. Acesso em 17 ago. 2009. doi:10.1111/j.1740-8261.2007.00252.x.

NAKAYAMA, H. et al. Correlation of cardiac enlargement as assessed by vertebral heart size and echocardiographic and electrocardiographic findings in dogs with evolving cardiomegaly due to rapid ventricular pacing. Journal Veternary Internal Medicine, v.15, p.217-221, 2001. Disponível em: <http:// onlinelibrary.wiley.com/doi/10.1111/j.1939-1676.2001.tb02314.x>. Acesso 17 ago. 2009. doi: 10.1111/j.1939-1676.2001.tb02314.x.

ROOT, C.R.; BAHR, R.J. The heart and great vessels. In THRALL, D.E. Textbook of veterinary diagnostic radiology. 4.ed. Philadelphia: Saunders, 2002. p.402-419.

SILVERMAN, S.; SUTTER, P.F. Influence of inspiration and expiration on canine thoracic radiographs. Journal of American Veterinary Medical Association. v.166, p.502$510,1975$.

SLEEPER, M.M.; BUCHANAN, J.W. Vertebral scale system to measure heart size in growing puppies. Journal of American Veterinary Medical Association, v.219, n.1, p.57-59, 2001.

SOARES, E.C. et al. Aspectos radiográficos da doença valvar crônica. Ciência Rural, v.34, p.119-124, 2004. Disponível em: http://www.scielo.br/pdf/cr/v34n1/a18v34n1.pdf. Acesso 06 de dezembro 2009.

TOAL. R.L. et al. Influence of cardiac cycle on the radiographic appearance of the feline heart. Veterinary Radiology \& Ultrasound, v.26, p.63-69, 1985. Disponível em: <http://onlinelibrary.wiley.com/ doi/10.1111/j.1740-8261.1985.tb01118.x>. Acesso 17 ago. 2009. doi: 10.1111/j.1740-8261.1985.tb01118.x. 